\title{
EARTHQUAKE FORECAST BY TIME-DEPENDENT HAZARD MODELS
}

\author{
Console R. ${ }^{1,2}$ \\ ${ }^{1}$ Center of Integrated Geomorphology for the Mediterranean Area, 85100, Potenza, Italy, \\ r.console@cgiam.org \\ ${ }^{2}$ Istituto Nazionale di Geofisica e Vulcanologia, 00143, Roma, Italy, rodolfo.console@ingv.it
}

\begin{abstract}
This presentation outlines methodological aspects of earthquake forecasting. The recurring debates concerning predictability of earthquakes clearly show how this problem is centred on the difficulty of systematically testing the numerous methodologies that in the years have been proposed and sustained by the supporters of prediction. This difficulty starts, sometimes, from the lack of a quantitative and rigorous definition of the concerned precursor, and other times from the lack of continuous observations, upon which statistical analyses could be based.

After an introduction concerning the definition of earthquake precursors, the way how to validate forecast hypotheses and the cost associated to their operational application, I give two examples of time-dependent hazard models, for long-term and short-term earthquake forecasts respectively.

Considering the long-term forecast modelling, the effect of stress change due to previous historical earthquakes on the probability of occurrence of future earthquakes on neighbouring faults is taken into account. Following a standard methodology developed a couple of decades ago, the probability of occurrence in the next 50 years for a characteristic earthquake on known seismogenic structures can be estimated by a time-dependent renewal model. Then, a physical model for the Coulomb stress change caused by previous earthquakes on these structures is applied. The influence of this stress change on the occurrence rate of characteristic earthquakes is computed taking into account the permanent perturbation (clock advance). The method so developed is applied to the computation of earthquake hazard of the main seismogenic structures recognized in the Southern Apennines region, for which both historical and paleoseismological data are available.

A popular short-term time-dependent hazard forecast model is the epidemic model. In this model earthquakes are regarded as the realization of a stochastic point process, and their magnitude distribution is described by the Gutenberg-Richter law with a constant b-value. The occurrence rate density is computed by the sum of two terms, one representing the independent, or spontaneous activity, and the other representing the activity induced by previous earthquakes. While the first term depends only on space, the second one is factored into three terms that respectively include the magnitude, time and location of the past earthquakes. An example of application of the epidemic model to the 2009 L'Aquila seismic series is shown.
\end{abstract}

Keywords: Precursors, Earthquake forecast, time-dependent hazard. 


\section{Introduction}

Among a wide category of natural hazards, earthquakes are regarded with particular concern because events of high intensity hit a large number of people at the same time and in a wide geographic area, often without any kind a warning to be used for the safety of lives and properties. In this respect, earthquake prediction has attracted the interest of seismologists since the early times of this science.

During the 70's, observations on earthquake precursors were so frequently reported in literature, that the opinion that earthquake prediction could have become substantially achievable in relatively short time was predominant within the seismological community. However, in spite of the significant progress obtained in seismology during the last decades, the optimism about the possible implementation of earthquake disaster countermeasures based on earthquake prediction has dramatically dropped down.

The situation concerning earthquake prediction studies has been characterised by a large variety of opinions: they range from those people who were quite confident in being able to predict all earthquakes (Varotsos et al., 1996), to those who were saying that earthquake prediction is intrinsically impossible (Geller et al., 1997). The possibility of coexistence of such different ideas largely comes from the lack of a common understanding on how prediction should be defined and its validity should be tested.

In the latest decade the term "earthquake prediction" has been more and more often substituted by the term "earthquake forecast", by means of which seismologists intend a quantitative definition of the probability that an earthquake in a specified magnitude range, geographic area and time interval will occur. A quantitative definition of the probability of occurrence admits also quantitative tests and comparisons among different forecasting models. In analogy with other geophysical phenomena, earthquakes are considered to behave as a kind of self-organized criticality (SOC) systems for which a deterministic approach is impossible, but are suitable for a sort of statistical forecast, such as "weather forecast" in meteorology. In this respect, the term "operational forecast" has become popular in the most recent years (Jordan et al., 2011).

The IASPEI Sub-Commission for Earthquake Prediction defined a precursor as "a quantitatively measurable change in an environmental parameter that occurs before mainshocks, and that is thought to be linked to the preparation process for this mainshock" (Wyss, 1991). In the context of this presentation, the set of ideas that are the basis and lead to the quantitative definition of a precursor is called a "hypothesis", or a "model". For a statistical test of a hypothesis, it is not necessary that it has an a-priori justification: even an empirical method is suitable for a rigorous a-posteriori evaluation. However, it has to be stressed that the hypothesis, or the model, characterising the concerned anomaly or precursor, should be defined in univocal way, so as it could be objectively recognised and evaluated in any circumstance and by any observer.

The wide variety of precursors proposed in the past literature is usually distinguished in three main categories, according to the range of time delays by which the initial observation of a the precursors is followed by the occurrence of the target event; namely, tens of hundreds of years for long-term precursors, a few months to years for medium-term precursors and hours to days for short-term precursors. In the following, examples of long- and short-term precursors will be considered.

\section{Simple statistical analysis of forecasts}

The simplest scenario in which we may consider a model of earthquake prediction is represented in Fig. 1. In this 3-dimensional space, the total volume of analysis $V_{T}$, given by the product of the geographical area by the time spanned by the observations, is called target volume. The earthquake occurrences (E) are represented as points characterised by their spatial co-ordinates, and origin time, for the events that exceed a given magnitude threshold. So, the magnitude of the events, that constitutes the fourth most common parameter contained in a usual catalogue, is a critical parameter 
in the representation of Fig. 1. The observation of a precursor is also represented by one point, defined by its location and occurrence time, and it generates an alarm (A). The volume in which an earthquake related to that precursor is expected (alarm volume) is a well-defined cylinder of given radius $(\mathrm{R})$ and duration $(\mathrm{T})$, depending on the type and the past experience had with the precursor. $\mathrm{R}$ and $\mathrm{T}$ may, in general, change for the various alarms. In case they don't change, we may define the alarm volume $V_{A}$ as constant for all the alarms. The prediction related to the occurrence of a precursor (or a set of precursors) is that an earthquake of magnitude equal to or larger than a given threshold magnitude (target event) will occur in the concerned alarm volume. If an earthquake really occurs in the alarm volume, it is called a success $(\mathrm{S})$. If it occurs outside of any alarm volume, it is called a failure of predicting (FP). An alarm that is not associated to any target earthquake is called a false alarm (FA).

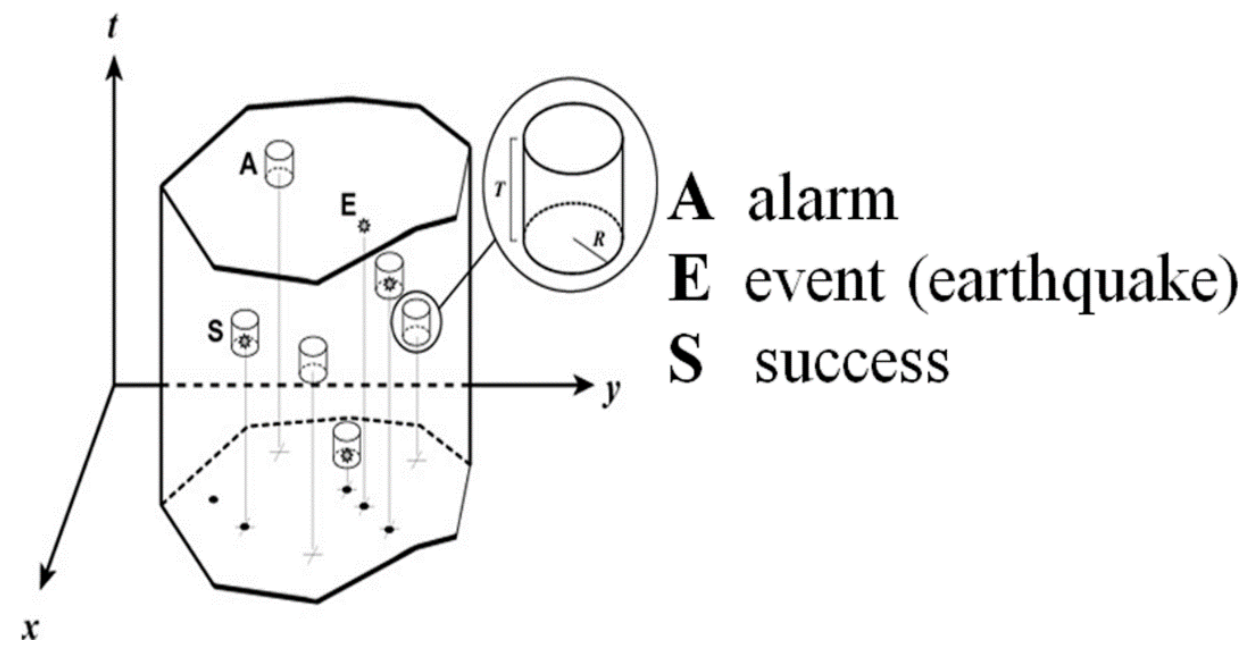

Figure 1 - Three-dimensional environment in which earthquakes and alarms are located.

The $x$ and $y$ axes are the geographical co-ordinates of a given seismic area. The vertical axis represents time increasing from the bottom. The symbols have the following meaning: $E$ (earthquakes), A (alarms), $\mathrm{R}$ (alarm radius), $\mathrm{T}$ (alarm duration), $\mathrm{S}$ (successes). An alarm that doesn't contain an earthquake is a false alarm (FA), and an earthquake that occurs outside of any alarm is a failure of predicting (FP).

The analysis of the performance of a specific method of prediction is carried out easily, if the observation of a sufficient number of past cases is available. Let $N_{S}, N_{A}$ and $N_{E}$ be the number of success, the number of alarms and the total number of earthquakes observed in the target volume, respectively.

The following statistical parameters are commonly considered in earthquake prediction evaluation:

-Success rate $=N_{S} / N_{A}$ (the rate at which precursors are followed by target events in the related alarm volume);

-False alarm rate $=\left(N_{A}-N_{S}\right) / N_{A}=(1$ - success rate $)$ : the rate at which precursors are not followed by target events;

-Alarm rate $=N_{S} / N_{E}$ : the rate at which target events are preceded by precursors;

-Failure rate $=\left(N_{E}-N_{S}\right) / N_{E}=(1-$ alarm rate $)$ : the rate at which target events are not preceded by precursors; 
- Probability gain $=\left(N_{S} /\left(N_{A} V_{A}\right)\right) /\left(N_{E} / V_{T}\right)$ : the ratio between the rate at which target events occur in the alarm volume and the average rate at which target events occur over the whole target volume.

The search for a good prediction method should be aimed to improve both the success and the alarm rates. It is clear that these two requirements are often in conflict with each other: more numerous alarms will tend to make the alarm rate higher, but they will, in general, make the success rate lower. There is one trivial way to get both these quantities higher by giving few alarms over very large alarm volumes. In this case, a large fraction of alarms would be successful and a small fraction of earthquakes would be missed, but this would be obtained increasing the cost of alarms at unsustainable levels. The limit case of this trend would be to give a permanent alarm over the whole target area, so as to have all the earthquakes predicted and no false alarms.

The probability gain is often regarded as a parameter that allows a comparison between different hypotheses. In general, a method of prediction can be considered significant if it achieves a probability gain greater than one, but the use of this concept should be made with some care. In fact, having defined the target volume $V_{T}$ as the total volume of analysis, the value of the probability gain is conditioned by the fact that the spatial distribution of seismicity is not homogeneous. So, regardless of the occurrence time of the earthquakes, a prediction model can easily achieve probability gains greater than one simply by issuing alarms in the most active zones of the target region. In this regard, the computation of the probability gain should be performed limiting the geographical extension of the target volume to the sum of the areas of alarm only, still maintaining the time length equal to the total time of analysis. In this way, only the time dependence in probability would be influent on the result of the computation.

\section{Economical aspects of forecasting}

Even if a hypothesis of earthquake forecasting is statistically demonstrated to achieve values of probability gain larger than one, its practical usefulness could still be questionable in relation to the costs that the community should support for its real implementation. The problem of issuing or not an earthquake alarm has been considered as a problem of optimal decision making in the context of its economical implications. Vere-Jones (1995) gave a simple outline of the basic arguments, in light of which the following considerations are derived, with some modifications. The long-term cost $C_{t o t}$ that the community has to support in relation to earthquake damages is basically given by the sum of three terms:

where:

$$
C_{t o t}=\alpha l(A)+C_{p} N_{p}(A)+C_{u} N_{u}(A),
$$

$\alpha$ is the cost of maintaining an alarm per unit time,

$l(A)$ is the total length of alarms,

$C_{p}$ is the cost for recovering the damages after a predicted event,

$C_{u}$ is the cost for recovering the damages after an unpredicted event,

$N_{p}(A)$ is the number of predicted events,

$N_{u}(A)$ is the number of unpredicted events.

In equation (1), $A$ defines the level of protection that one can imagine to implement. It is related to the choice for the thresholds that the relevant parameters of the prediction model should exceed for having an alarm declared. Here the cost of operating the prediction system is assumed negligible.

$C_{p}$ and $C_{u}$ are considered, in a very rough approximation, constant. This is reasonable only if the intensity of all the earthquakes is the same. In this respect, equation (1) should be computed separately for each intensity class and each particular area, and the results summed accordingly. It is reasonable to assume always $C_{u}>C_{p}$ for the same class of intensity. However, the determination 
of their values implies a lot of engineering and economical studies, while the geophysical contribution is focused on the estimate of the relation between $l(A), N_{p}(A)$ and $N_{u}(A)$, for a given $A$.

Introducing the total number of earthquakes $N_{e}=N_{p}(A)+N_{u}(A)$, equation (6) can be written as:

$$
C_{\text {tot }}=C_{u} N_{e}+\alpha l(A)-\left(C_{u}-C_{p}\right) N_{p}(A),
$$

In the latter equation (2), the first term is the total cost that the community should pay to the earthquakes if no prediction system was operated. The second term is the additional cost to be supported for maintaining the alarms, and the third term is the cost saved by the community because of the successful predictions. Any prediction system should at least fulfil the requirement that the latter is larger than the former, so as $C_{t o t}<C_{u} N_{e}$. In this simplified view, the optimal strategy would be the adoption of the level of protection $A$ at which the total $\operatorname{cost} C_{t o t}$ is minimum. It should be noted, however, that a slight increase of the level of protection would yield more safety to people at nearly no additional cost (Figure 2).

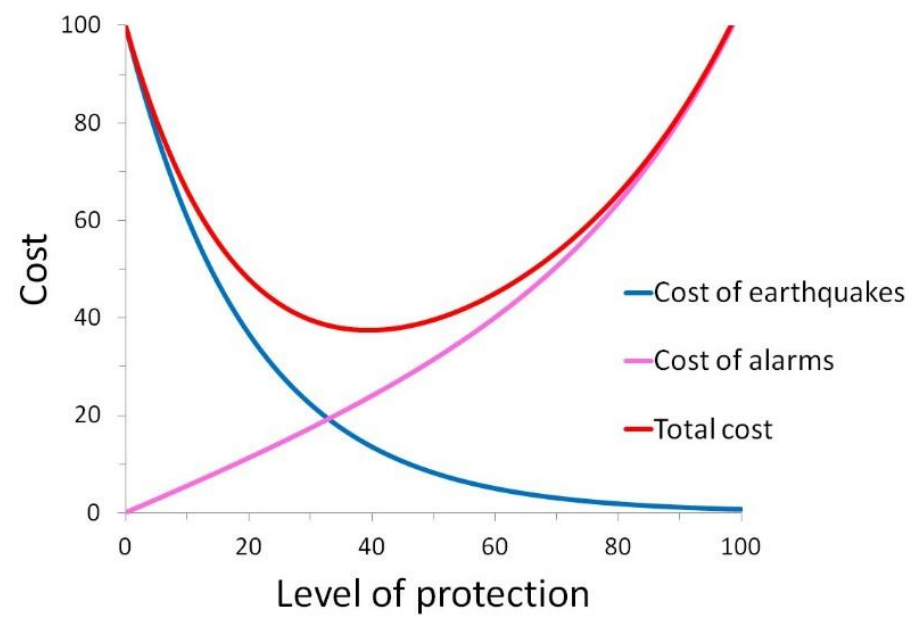

Figure 2 - Qualitative representation of long term costs that a community should pay in relation to damages produced by earthquakes, versus the level of protection adopted for issuing alarms. The scale in both $x$ and $y$ axes is intentionally arbitrary. The cost paid after the earthquakes for recovering damages ("cost of earthquakes") decreases monotonically with the level of protection, but it doesn't tend to zero because there are damages that cannot be avoided, even if the earthquake is predicted with large advance. The cost of maintaining earthquake alarms ("cost of alarms") is supposed to increase linearly with the level of protection. The total cost exhibits a minimum that corresponds to the most economical strategy.

\section{Long-Term time-dependent earthquake forecast}

A standard procedure for seismic hazard assessment assumes that all relevant earthquakes occur on well recognized faults with characteristic mechanism and size. The procedure needs the adoption of a probability density function $f(t)$ (pdf) for the inter-event time between consecutive events on each fault, together with some basic parameters of the model. One can adopt either a time independent Poisson model or a renewal model. For the former model, the expected recurrence time $T_{r}$ is the only necessary piece of information. For the latter, also a parameter as the coefficient of variation (also known as aperiodicity) $\alpha$ of the inter-event times is required. 
We adopt the Brownian Passage Time (BPT) distribution introduced by Matthews et al. [2002] to represent the inter-event time probability distribution for earthquakes on single sources in Italy. This distribution is expressed as

$$
f\left(t ; T_{r}, \alpha\right)=\left(\frac{T_{r}}{2 \pi \alpha^{2} t^{3}}\right)^{1 / 2} \exp \left\{-\frac{\left(t-T_{r}\right)^{2}}{2 T_{r} \alpha^{2} t}\right\} .
$$

Earthquake probability may be either increased or decreased with respect to what would be expected by a simple renewal model. The interaction is taken into consideration by the computation of the Coulomb static stress change or the Coulomb Failure Function $(\Delta \mathrm{CFF})$ caused by previous earthquakes on the concerned fault [King et al., 1994]:

$$
\Delta C F F=\Delta \tau+\mu^{\prime} \Delta \sigma_{n},
$$

where $\Delta \tau$ is the shear stress change on a given fault plane (positive in the direction of fault slip), $\Delta \sigma_{n}$ is the fault-normal stress change (positive when unclamped), and $\mu^{\prime}$ is the effective coefficient of friction.

As $\triangle C F F$ is strongly variable in space, we consider its value in the point of the triggered fault where it may have the largest effect. For this computation, the knowledge of the fault parameters (strike, dip, rake, dimensions, average slip) is necessary for both triggering and triggered sources.

The influence of $\triangle C F F$ on the probability for the future characteristic event is considered in two ways [Stein et al., 1997]. The first (permanent effect) assumes that the time elapsed since the previous earthquake is modified from $t$ to $t^{\prime}$ by a shift proportional to $\triangle C F F$, that is

$$
t^{\prime}=t+\frac{\Delta C F F}{\dot{\tau}}
$$

where $\dot{\tau}$ is the tectonic stressing rate.

The expected number of events $N$ over a given time interval $(t, t+\Delta t)$ is computed by integration. Under the hypothesis of a generalized Poisson process, we may estimate the probability of occurrence for the earthquake in the given time interval:

$$
P=1-\exp (-N)
$$

where $\quad N=\int_{t}^{t+\Delta t} R(t) d t$

For an application of the above mentioned method, we make use of the most comprehensive source of information available about Italian seismogenic sources: the Database of Individual Seismogenic Sources (DISS) owned by Istituto Nazionale di Geofisica [DISS Working Group, 2006]. For each source, DISS stores, among others, the following parameters, estimated from various kinds of geological, geodetic, geomorphological and seismological data, or inferred from other parameters through physical relationships:

- Location (lat/long) of the centre of the fault

- Length and width of the fault

- Minimum and maximum depth

- Strike, dip and rake of the fault

- Average slip 
- Slip rate (minimum and maximum)

- Recurrence time (minimum and maximum)

- Maximum magnitude (Mw)

- Date of the latest earthquake

Table 1 - Parameters of two seismogenic structures reported by DISS in the study area of Central-Southern Apennines.

\begin{tabular}{|c|c|c|}
\hline & Sulmona basin & Melandro-Pergola \\
\hline Date of latest event & 1315.12 .3 & 1857.12 .16 \\
\hline Slip rate $(\mathrm{mm})$ & $0.24 \pm 0.06$ & $0.11 \pm 0.04$ \\
\hline Recurrence time (years) & $2300 \pm 1370$ & $4300 \pm 3700$ \\
\hline $\begin{array}{l}\text { Max. Poisson probability for } \\
\text { the next } 50 \text { years }\end{array}$ & $5.3 \%$ & $8.8 \%$ \\
\hline Elapsed time (years) & 692 & 150 \\
\hline $\begin{array}{l}\text { Max. renewal probability for } \\
\text { the next } 50 \text { years }\end{array}$ & $8.4 \%$ & $1.8 \%$ \\
\hline Max. $\triangle \mathrm{CFF}(\mathrm{Mpa})$ & 0.15 & 0.19 \\
\hline $\operatorname{Max} . \Delta \mathrm{t}($ years $)$ & 132 & 394 \\
\hline $\begin{array}{l}\text { Max. modified probability } \\
\text { for the next } 50 \text { years }\end{array}$ & $11.8 \%$ & $18.6 \%$ \\
\hline
\end{tabular}

Two sources of the Apennines chain have been considered for an application of the above mentioned method: the Sulmona Basin and the Melandro-Pergola faults. They are respectively characterized by a time elapsed since the latest characteristic earthquake comparable and much shorter than the average recurrence time. As shown in Table 1, the probability of occurrence of a new characteristic earthquake, computed through the time-independent Poisson model in the next 50 years, is dramatically affected by both the value obtained for the stress change CFF (shown in Figure 3 and 4) and the elapsed time. 


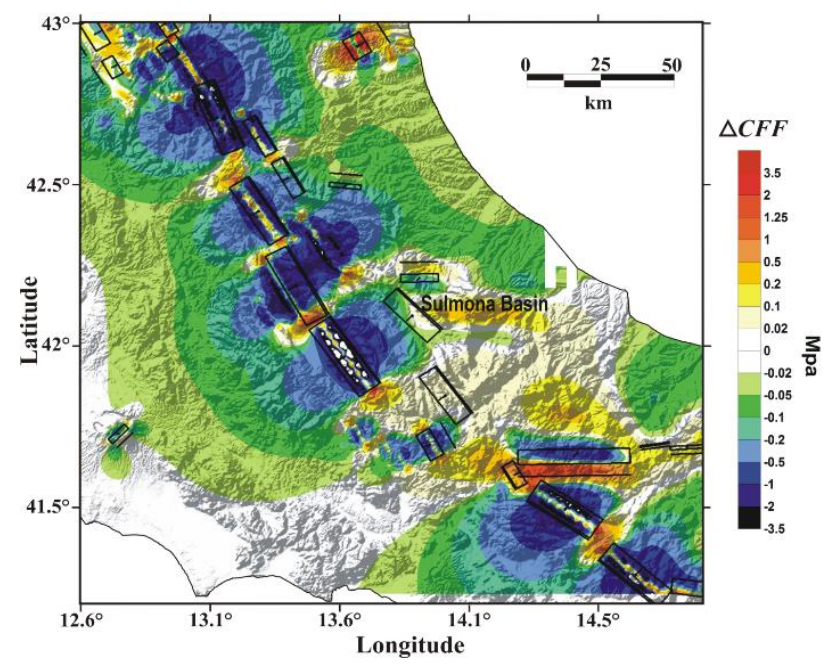

Figure 3 -- Map of the Coulomb Stress changes $\triangle C F F$ cumulatively released by all the earthquakes following the 1315 (Sulmona basin) earthquake. $\triangle C F F$ is computed on the basis of the fault mechanism of the concerned earthquake.

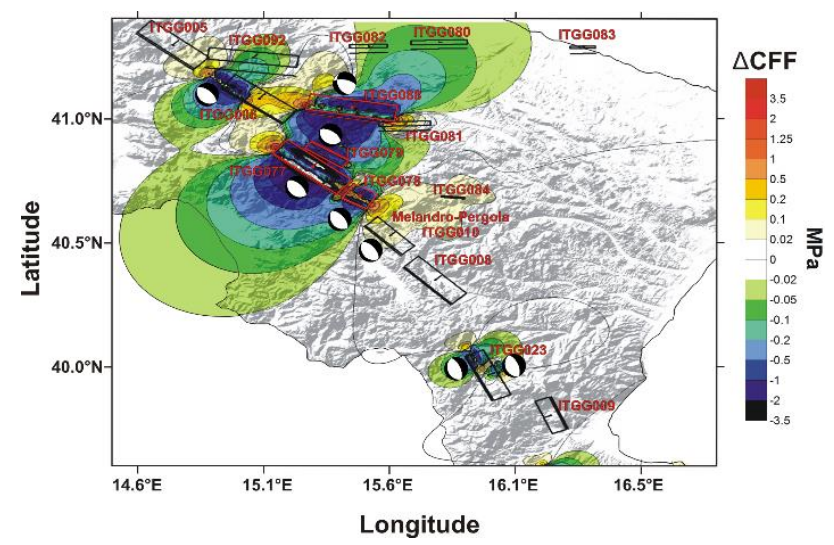

Figure 4 - Map of the Coulomb Stress changes $\triangle C F F$ cumulatively released by all the earthquakes following the 1857 (Melandro-Pergola) earthquake. $\triangle C F F$ is computed on the basis of the fault mechanism of the concerned earthquake.

\section{Short-term earthquake forecasting}

Here we give a brief outline of the method for modeling the interrelation of any earthquake with any other. Following Ogata (1998), Console and Murru (2001), Console et al. (2003), earthquakes are regarded as the realization of a stochastic point process. The occurrence rate density, at any instant of time and geographical point, is computed by the contribution of every previous events using a kernel function that takes in proper account: (a) the magnitude of the triggering earthquake, (b) the spatial distance from the triggering event, and (c) the time interval between the triggering event and the instant considered for the computation. The magnitude distribution adopted here is the 
Gutenberg-Richter law with a constant $b$-value. The occurrence rate density of earthquakes in space and time triggered by previous earthquakes can be described as:

$$
\lambda(x, y, t, m)=f_{r} \cdot \lambda_{0}(x, y, m)+\sum_{i=1}^{N} H\left(t-t_{i}\right) \cdot \lambda_{i}(x, y, t, m)
$$

where $f_{r}$ is a factor called the 'failure rate' (i.e. the ratio between the expected number of independent events and the total number of events), $\lambda_{0}(x, y, m)$ represents the time-invariant background seismicity, obtained from the analyzed catalog by means of a smoothing algorithm [Console and Murru, 2001]; $t_{i}$ is the occurrence time of the $N$ earthquakes; $H(t)$ is the step function, and $\lambda_{i}(x, y, t, m)$ is the single contribution of the previous earthquakes. The first and the second term on the right hand side of equation (1) represent the time-invariant "spontaneous" and the time-varying "triggered" seismicity, respectively. The rate density corresponding to any earthquake is the sum of these two components.

We assume a spatial distribution represented by an isotropic function and the time dependence as the modified Omori law:

$$
\lambda_{i}(x, y, t, m)=k \cdot\left(t-t_{i}+c\right)^{-p} \cdot\left[d_{i}^{2} /\left(r^{2}+d_{i}^{2}\right)\right]^{q} \cdot 10^{-b\left(m-m_{0}\right)}
$$

where $r$ is the distance from $(x, y)$ to $\left(x_{i}, y_{i}\right)$ and $d_{\mathrm{i}}$ is equal to $d_{0} 10^{\left(m_{\mathrm{i}}-m_{0}\right) / 2}$ so that the average triggering distance of the aftershock zone is proportional to the square root of the main shock rupture area, as observed in real data [Kagan, 2002]. In this way $d_{i}$ depends on the magnitude of the triggering event. The free parameter $d_{0}$ controls the shape of the distribution. The $q$ parameter of the space distribution of triggered events is fixed to 1.5 , for consistency with the theory of elasticity when $r \rightarrow \infty$.

The set of free parameters of the epidemic model is usually obtained by applying the algorithm to a learning data set and fitting the parameters by means of the maximum likelihood criterion. The algorithm was recently applied in forward retrospective way to the seismic activity preceding and following the Mw6.3 L'Aquila earthquake of 6 April 2009 (Murru et al., 2015). For that study case the best fit was carried out exclusively by a learning dataset preceding the occurrence of the mainshock. After the learning phase, the algorithm can be applied to for testing purposes in retrospective way (see .e.g. Table 2), or in real time for short-term earthquake forecast in operational way.

Table 2 - Probability of an earthquake of magnitude $\geq 5$ in 24 hours in the area of L'Aquila (ETAS model)

$\begin{array}{ll}\text { 1 December 2008 } & 0.02 \% \\ \text { 27 March 2009 } & 0.06 \% \\ \text { 31 March 2009 } & 1.5 \% \\ \text { 5 April 2009 22:00 } & 0.4 \% \\ \text { 5 April 2009 23:00 } & 1.6 \% \\ \text { 6 April 2009 01:00 } & 2.0 \% \\ \text { 6 April 2009 04:00 } & 8.3 \% \\ \text { 6 April 2009 12:00 } & 12.8 \% \\ \text { 20 April 2009 } & 7.6 \% \\ \text { 10 May 2009 } & 4.4 \%\end{array}$

\section{References}

Console, R. and Murru, M., 2001. A simple and testable model for earthquake clustering, J. Geophys. Res., 106, 8699-8711.

Console, R., Murru, M. and Lombardi, A.M., 2003. Refining earthquake clustering models, J.Geop hys. Res., 108, 2468, doi: 10.1029/2002JB002130. 
Geller, R.J., Jackson, D.D., Kagan, Y.Y. and Mulargia, F., 1997. Earthquakes cannot be predicted. Science, 275, 1616-1617.

Jordan, T.H. et al., 2011. Operational earthquake forecasting. State of Knowledge and Guidelines for Utilization, Annals of Geophysics, 54, 4, 2011, doi: 10.4401/ag-5350.

Kagan, Y.Y., 2002. Aftershock zone scaling, Bull. Seismol. Soc. Amer., 92(2), 641-655.

King, G.C.P., Stein, R.S. and Lin, J., 1994. Static stress changes and the triggering of earthquakes, Bull. Seismol. Soc. Am., 84, 935-953, 1994.

Matthews, M.V., Ellsworth, W.L. and Reasenberg, P.A., 2002. A Brownian model for recurrent earthquakes, Bull. Seism. Soc. Am., 92, 2233-2250.

Murru, M., Zhuang, J., Console, R. and Falcone, G., 2015. Short-term earthquake forecasting experiment before and during the L'Aquila (central Italy) seismic sequence of April 2009, accepted by Annals of Geoph.

Ogata, Y., 1998. Space-time point-process models for earthquake occurrences, Ann. Inst. Statist. Math., 50, 379-402.

Stein, R., Barka, A.and Dieterich, J., 1997. Progressive failure on the North Anatolian fault since 1939 by earthquake stress triggering, Geophys. J. Int., 128, 594-604

Varotsos, P. et al., 1996. In A Critical Review of VAN, J. Lighthill, Ed., World Scientific, Singapore, $29-76$.

Vere-Jones, D., 1995. Forecasting earthquakes and earthquake risk. Int. J. of Forecasting, 11, 503538.

Wyss, M., ed., 1991. Evaluation of Proposed Earthquake Precursors, American Geophysical Union, $94 \mathrm{pp}$. 